\title{
Volatilidade de dados intradiários: comportamento multiescala do Ibovespa frente à pandemia COVID-19
}

\section{Volatility of intraday financial data: Multiscale Ibovespa behavior under to the COVID-19 pandemic}

\author{
Marcela de Marillac Carvalho ${ }^{1}$; Luiz Otávio de Oliveira Pala ${ }^{2}$; \\ Thelma Sáfadi ${ }^{3}$
}

\begin{abstract}
Resumo
Em mercados financeiros, a modelagem da volatilidade vem sendo uma estratégia muito utilizada por refletir as incertezas sobre as variações dos preços dos ativos. Incorporando peculiaridades de séries financeiras, este estudo estimou a volatilidade para o índice intradiário do mercado acionário brasileiro (Ibovespa) por meio de modelos ARIMA-APARCH em diferentes frequências temporais com o auxílio da técnica de decomposição wavelet MODWT. Este trabalho propõe a análise dos impactos dos componentes de frequência no comportamento da volatilidade de retornos intradiários com o uso de séries de detalhes wavelet em diferentes horizontes temporais, em um período atípico nos mercados financeiros mundiais, gerado pela pandemia do COVID-19. Os resultados empíricos sugerem baixa volatilidade incondicional e fortes sinais de persistência em todas as frequências analisadas. A assimetria na volatilidade é evidenciada nas frequências maiores, com efeito alavancagem presente apenas nas séries de detalhes com variações de 15-120 min., o que é corroborado com os resultados obtidos com a série reconstruída. Os comportamentos evidenciados impactam na elaboração de estratégias de investimento de curto prazo e gerenciamento de risco, uma vez que os choques positivos e negativos, como os dados pela pandemia mundial do COVID-19, têm impactos diferenciados sobre a volatilidade dos retornos em prazos menores. As informações obtidas podem contribuir na análise de futuros eventos atípicos no mercado acionário brasileiro embasando a tomada de decisão dos agentes econômicos.
\end{abstract}

Palavras-chave: Volatilidade. Índice Ibovespa. Modelos ARIMA-APARCH. Transformada de Wavelets.

\begin{abstract}
In financial markets, volatility modeling has been a strategy widely used because it reflects uncertainties about changes in asset prices. Incorporating peculiarities of financial series, this study estimated the volatility for the intraday index of the Brazilian stock market (Ibovespa) using ARIMA-APARCH models in different time frequencies with the aid of the wavelet MODWT decomposition technique. This work proposes an analysis of the impacts of the frequency components on the behavior of the volatility of intraday returns using the series of details wavelet in different time horizons, in an atypical period in the global financial markets, generated by the COVID-19 pandemic. The empirical results suggest low unconditional volatility and strong signs of persistence in all analyzed frequencies. The asymmetry in volatility is evidenced in the higher frequencies, the leverage effect being present only in the series of details with variations of 15-120 min., which is corroborated with the results obtained with the reconstructed series. The evidenced behaviors have an impact on the elaboration of short-term investment strategies and risk management, since the positive and negative shocks, such as those given by the world pandemic of COVID-19, have different impacts on the volatility of returns in shorter periods. The information obtained can contribute to the analysis of future atypical events in the Brazilian stock market, supporting the decision-making of economic agents.
\end{abstract}

Keywords: Volatility. Ibovespa index. ARIMA-APARCH models. Wavelet transform.

\footnotetext{
${ }^{1}$ Doutoranda em Estatística e Exp. Agro., Dept. de Estat., UFLA, Lavras, MG, Brasil; E-mail: marcela.carvalho2@estudante.ufla.br

2 Doutorando em Estatística e Exp. Agro., Dept. de Estat., UFLA, Lavras, MG, Brasil; E-mail: luiz.pala@ estudante.ufla.br

${ }^{3}$ Prof $^{a}$. Dr ${ }^{\mathrm{a}}$., Deptartamento de Estatística, UFLA, Lavras, MG, Brasil; E-mail: safadi@ des.ufla.br
} 


\section{Introdução}

A análise de séries intradiárias de mercados financeiros apresenta desafios específicos. Estes dados incluem componentes de alta frequência, variantes ao longo do tempo, caracterizados por dinâmicas complexas que retratam os frequentes movimentos de preços de ativos em um dado período de negociação (GALLEGATI; SEMMLER, 2014; IN; KIM, 2013). Os retornos financeiros apresentam peculiaridades (stylized facts) como:

i) alto valor de curtose;

ii) enômenos de alta volatilidade que se aglomeram no tempo;

iii) presença de padrões sazonais durante o período de negociação dos ativos, dentre outras (MORETTIN, 2017).

No que se refere a estas questões, em finanças, a volatilidade é um dos componentes mais relevantes. Essa medida corresponde ao desvio padrão condicional dos retornos dos ativos, que pode se manifestar de várias maneiras numa série financeira, refletindo as incertezas sobre as variações dos preços. Quanto maior a variação do preço de um ativo ao longo de um período de tempo, mais volátil ele é considerado. Aspectos associados à volatilidade do mercado como presença de persistência e assimetria aos choques de informação em séries de retornos financeiros tem sido um importante objeto de estudos empíricos em Audrino e Hu (2016), Baur e Dimpfl (2018), Pan e Liu (2018), Patton e Sheppard (2015), Ramzan, Ramzan e Zahid (2012), dentre outros.

A complexidade intrínseca destes sistemas demanda metodologias que incorporem na modelagem da volatilidade essas características tão comuns nas séries financeiras intradiárias. Para tanto, modelos de heterocedasticidade condicional da família ARCH (Autoregressive Conditional Heteroskedasticity), introduzidos a partir de Engle (1982), vêm se mostrando uma importante ferramenta em áreas como precificação de ativos, seleção de portfólios e avaliação de riscos por incorporar problemas como distribuição assimétrica, persistência e efeito alavancagem, ver em Black (1976).

Um dos modelos desta classe é o APARCH (Asymmetric Power ARCH), desenvolvido por Ding, Granger e Engle (1993). Além de refletir a persistência, este modelo adiciona a flexibilidade de um expoente do desvio padrão condicional variando com um coeficiente de assimetria para considerar o efeito de alavancagem que decorre da característica de aversão ao risco inerente aos agentes econômicos. Conforme Daly (2008), o fenômeno de alavancagem surge quando em momentos de baixa nos preços a volatilidade de seu retorno aumenta, enquanto, em períodos de alta nos preços a volatilidade não é tão intensa. $\mathrm{O}$ APARCH ainda inclui sete outras extensões ARCH como casos especiais: ARCH e GARCH clássicos, GJRGARCH, TS-GARCH, TARCH, NARCH e LogARCH (ou MGARCH).

Essas informações específicas associadas a volatilidade podem ser analisadas em termos da decomposição de escalas de variação temporal. Hasbrouck (2016) e Nava, Matteo e Aste (1989) evidenciam que considerar diferentes escalas de tempo contribui para explicar a complexidade inerente dos comportamentos da volatilidade de índices financeiros, captando efeitos de padrões periódicos intradiários e de calendário refletidos nos movimentos dos preços (LATIF et al., 2011; ROSSI, 2015) e impactos de tendências de longo ou curto prazo (GALLEGATI; SEMMLER, 2014). Neste contexto, o uso de técnicas wavelet vem ganhando muitos adeptos (BIAGE, 2019; JENSEN; WHITCHER, 2014; OMANEADJEPONG; ABABIO; ALAGIDEDE, 2019; SHAH; TALI; FAROOQ, 2018).

Este estudo busca compreender as implicações da microestrutura financeira na volatilidade, analisando os impactos de diferentes frequências de tempo sobre o comportamento da volatilidade intradiária, aplicando o processo ARIMA-APARCH para estimar a variância condicional em escalas a partir da decomposição wavelet da série temporal intradiária dos retornos. Essa técnica permite captar diferentes níveis de detalhes da série original de forma não agregada no tempo. O objetivo é analisar em cada frequência considerada aspectos como assimetria, variância incondicional, a presença da persistência e do efeito alavancagem no cenário em que incorre efeitos dos desdobramentos da pandemia do COVID-19 nos mercados financeiros mundiais.

Para a análise foram selecionados os retornos intradiários do índice Ibovespa, em um período que retrata os impactos do COVID-19. O Ibovespa corresponde ao indicador de desempenho médio de ações negociadas no mercado de capitais brasileiro e é um dos principais mercados da América Latina. Dessa forma, a contribuição deste estudo reside na avaliação dos impactos de diferentes ciclos financeiros nas estimativas da variância condicional da bolsa de valores brasileira, refletindo a forma com que importantes ações respondem aos efeitos da volatilidade em diferentes horizontes temporais. Além disso, por ser um 
mercado financeiro mais suscetível aos efeitos de crises, dado o período de amostragem, os resultados do trabalho podem ser utilizados futuramente na incidência de eventos de incertezas, embasando a gestão de investimentos de agentes econômicos.

O período de análise compreende entre 17 de março de 2020 a 11 de setembro de 2020, com intervalo de amostragem de 15 minutos, considerando um dia útil com 7 horas de negociação no mercado brasileiro. Propõe-se o uso da técnica de transformação de $w a$ velet discreta de sobreposição máxima (MODWT) de Percival e Walden (2000) que permite filtrar séries em diferentes componentes de frequência, preservando a variação da série original com invariância de deslocamento. Por apresentar propriedades importantes na análise multirresolução, a decomposição foi realizada usando o filtro wavelet (DAUBECHIES, 1992) com 2 momentos nulos.

\section{Estrutura e procedimentos metodológicos}

A seguir é apresentada a estrutura base dos procedimentos metodológicos utilizados neste estudo relacionados aos processos heterocedásticos condicionais e o modelo APARCH, bem como os aspectos sobre a decomposição MODWT. Os resultados foram obtidos com o auxílio do software $R$ (R CORE TEAM, 2020) utilizando os pacotes fGarch (WUERTZ et al., 2019) e wmtsa (CONSTANTINE; PERCIVAL, 2017).

\section{Dados}

Os dados das cotações do indicador Ibovespa foram obtidos no pacote QuantTools (KOVALEVSKY, 2018) em que cada registro contém informações sobre preço, volume negociado, dia e hora da negociação. A Tabela 1 contém a participação relativa das principais ações que compõem o índice. $\mathrm{O}$ período de análise abrange 17 de março de 2020 a 11 de setembro de 2020, com $n=3660$ observações. O intervalo de amostragem considerado foi de 15 minutos com tempo espaçado para as sete horas de negociação contínua no mercado brasileiro. A série analisada refere-se aos log-retornos intradiários $r_{d, m}=\ln \left(p_{d, m}\right)-\ln \left(p_{d, m-1}\right)$, em que $p_{d, m}$ é a cotação do ativo no período $m=1, \ldots, 28$ do dia útil $d=1, \ldots, 124$. A notação $r_{t}$ foi usada aqui para a série intradiária de retornos do Ibovespa $r_{d, m}$.

\section{Análise Wavelet}

A transformação de wavelet discreta de sobreposição máxima (MODWT) consiste na filtragem de
Tabela 1 - Principais ações que compõem a Carteira Teórica do Ibovespa para o perído de março a dezembro de 2020 .

\begin{tabular}{ccc}
\hline S/A & Código da Ação & Participação \\
\hline Vale do Rio Doce & VALE3 & $10,46 \%$ \\
Itaú Unibanco & ITUB4 & $6,38 \%$ \\
Petrobras & PETR4 & $5,62 \%$ \\
B3 & B3SA3 & $5,33 \%$ \\
Bradesco & BBDC4 & $5,09 \%$ \\
Petrobras & PETR3 & $4,40 \%$ \\
Magazine Luiza & MGLU3 & $3,19 \%$ \\
Ambev & ABEV3 & $2,95 \%$ \\
Banco do Brasil & BBAS3 & $2,34 \%$ \\
Weg & WEGE3 & $2,34 \%$ \\
Intermédica & GNDI3 & $2,33 \%$ \\
Lojas Renner & LREN3 & $1,95 \%$ \\
Natura & NTCO3 & $1,91 \%$ \\
Suzano & SUZB3 & $1,85 \%$ \\
JBS & JBSS3 & $1,84 \%$ \\
\hline
\end{tabular}

Fonte: Os autores.

uma série de tempo em informações multiescala. A MODWT apresenta propriedades essenciais na decomposição de séries temporais: é não ortogonal e invariante a translação, conservando a variação da série original, para mais detalhes ver Percival e Walden (2000). Dada essas características, é possível estimar a volatilidade usando séries reconstruídas das decomposições.

Para gerar as séries financeiras wavelet é necessário uma filtragem sucessiva nos dados de retornos. Para todos os inteiros $n$, de comprimento $L$, os filtros passa-baixa $\left\{g_{j, l}\right\}$ e passa-alta $\left\{h_{j, l}\right\}$, são aplicados para decompor um sinal e precisam atender aos seguintes critérios:

i) $\sum_{l=0}^{L-1} h_{l}=0$;

ii) $\sum_{l=0}^{L-1} h_{l}^{2}=\frac{1}{2}$;

iii) $\sum_{i=0}^{L-1} h_{i} h_{i+2 n}=0$;

iv) $g_{l}=-1^{l} h_{l-}$

Os filtros da MODWT são expressos como um dimensionamento dos filtros $\left\{g_{j, l}\right\}$ e $\left\{h_{j, l}\right\}$ da seguinte forma: $\widetilde{h}_{j, l}=h_{j, l} / 2^{j}$ e $\widetilde{g}_{j, l}=g_{j, l} / 2^{j}$. Com a vantagem de suporte compacto e ortogonalidade, neste trabalho foram empregados os filtros wavelet Daubechie $h_{l, j}=(-1)^{l-L_{j}} g_{L_{j}-1-l}$ com 2 momentos nulos $(d b 2)$ (DAUBECHIES, 1992).

Com entrada inicial dada por $\widetilde{s}_{0, t}=r_{t=0}^{N-1}$, o processo de decomposição ocorre a partir do algoritmo piramidal de Mallat (1989) a partir dos relacionamentos apresentados 
nas equações (1) e (2)

$$
\widetilde{s}_{j, t} \equiv \sum_{l=0}^{L_{j}-1} \widetilde{g}_{j, l} r_{t-l} \bmod N
$$

e

$$
\widetilde{d}_{j, t} \equiv \sum_{l=0}^{L_{j}-1} \widetilde{h}_{j, l} r_{t-l} \bmod N
$$

em que $L_{j}=\left(2^{j}-1\right)(L-1)+1$ corresponde ao tamanho do filtro associado a cada escala $j, j=1, \ldots, J$ e $\bmod N$ é um operador de módulo. Nos níveis $j$ e no índice de tempo $t$ os coeficientes de escala $\widetilde{s}_{j, t}$ referem-se a aproximação de $r_{t}$ que capturam oscilações mais longas, e os coeficientes $\widetilde{d}_{j, t}$ captam oscilações como mudanças estruturais, representando o detalhamento de $r_{t}$.

Como a MODWT preserva a variação original da série de entrada, dada as relações apresentadas nas equações (1) e (2), uma reconstrução de $r_{t}$ pode ser obtida como apresentado na equação (3)

$$
r_{t}=\sum_{j=1}^{J} \widetilde{d}_{j, t}+\widetilde{s}_{J, t} .
$$

\section{Séries wavelet de detalhes}

Os coeficientes $\widetilde{d}_{j, t}$ foram nosso objeto de análise, pois consistem em séries temporais que descrevem $r_{t}$ em níveis cada vez mais grosseiros de resolução, de forma não agregada no tempo. Cada nível de detalhe corresponde a um ciclo, sendo que quanto maior o nível de decomposição, maior o intervalo de tempo do ciclo (CROWLEY, 2007). As séries $\widetilde{d}_{j, t}$ utilizadas são as que captam, aproximadamente, variações de minutos, hora, semana e mês. As frequências são mensuradas de acordo com a Tabela 2, conforme Crowley (2007), e tem como base um dia útil do mercado brasileiro.

Tabela 2 - Variação e interpretação dos componentes de decomposição nos níveis de variação dos log-retornos intradiários do índice Ibovespa

\begin{tabular}{cc}
\hline Nível & Frequência \\
\hline$\widetilde{d}_{1, t}$ & $15-30$ minutos de negociação \\
$\widetilde{d}_{3, t}$ & $60-120$ minutos de negociação \\
$\widetilde{d}_{6, t}$ & $8-16$ horas $\approx 1-2$ dias de negociação \\
$\widetilde{d}_{8, t}$ & $32-64$ horas $\approx 5-9$ dias de negociação \\
$\widetilde{d}_{10, t}$ & $128-256$ horas $\approx 18-37$ dias de negociação \\
\hline
\end{tabular}

Fonte: Os autores.

Como as variações semanais captadas por $\widetilde{d}_{8, t}$, apresentam influência de dias da semana, como os chamados efeitos segunda e sexta-feira, para ajuste da volatilidade é necessário neste nível isolar essas sazonalidades como em Alberg, Shalit e Yosef (2008), conforme as equações (4) e (5), dadas por

$$
\widetilde{d}_{8, t}=\alpha_{1} \mathrm{Mo}_{t}+\alpha_{2} \mathrm{Tu}_{t}+\alpha_{3} \mathrm{We}_{t}+\alpha_{4} \mathrm{Th}_{t}+\alpha_{5} \mathrm{Fr}_{t}+\delta_{t}
$$

$\left(\widetilde{d_{8, t}}-\hat{\vec{d}_{8, t}}\right)^{2}=\alpha_{1} \mathrm{Mo}_{t}+\alpha_{2} \mathrm{Tu}_{t}+\alpha_{3} \mathrm{We}_{t}+\alpha_{4} \mathrm{Th}_{t}+\alpha_{5} \mathrm{Fr}_{t}+\varepsilon_{t}$

em que $\mathrm{Mo}_{t}, \mathrm{Tu}_{t}, \mathrm{We}_{t}, \mathrm{Th}_{t}, \mathrm{Fr}_{t}$, são variáveis dummies representando os dias de semana e $\hat{\vec{d}}_{8, t}$ o valor predito com a equação (4). A série filtrada é dada a partir das estimativas da equação (5) como $\widetilde{d}_{8, t}=\frac{\left(\tilde{d}_{8, t}-\hat{\widetilde{d}}_{8, t}\right)}{\sqrt{\hat{\eta}}}$, sendo $\hat{\eta}$ o valor estimado de $\left(\widetilde{d}_{8, t}-\hat{\vec{d}}_{8, t}\right)^{2}$.

\section{Modelo APARCH}

Processos denominados da classe ARCH expressam, de modo geral, a componente de volatilidade (variância condicional) e uma componente de inovação comumente assumida como Gaussiana, t-Student ou extensão dessas distribuições. Dentro dessa classe, o modelo APARCH foi desenvolvido para introduzir flexibilidade de um expoente variável com o coeficiente de assimetria para detectar impactos assimétricos de choques sobre a volatilidade (efeito alavancagem) de séries de retornos financeiros.

A estrutura geral do modelo $\operatorname{APARCH}(p, q)$ para desvio padrão condicional $\left(\sigma_{t}\right)$ de $r_{t}$ é dada por $r_{t}=\mu+\varepsilon_{t}$, em que $\varepsilon_{t}=z_{t} \sigma_{t}$ e $z_{t} \sim \mathscr{D}_{v}(0,1)$, com $\sigma_{t}^{\delta}$ definido na equação (6)

$$
\sigma_{t}^{\delta}=\omega+\sum_{i=1}^{p} \alpha_{i}\left(\left|\varepsilon_{t-i}\right|-\gamma_{i} \varepsilon_{t-i}\right)^{\delta}+\sum_{j=1}^{q} \beta_{j} \sigma_{t-j}^{\delta}
$$

sujeito as seguintes restrições nos parâmetros para garantir que a variância condicional seja positiva e fracamente estacionária:

$$
\begin{aligned}
& \text { - } \omega \geq 0, \delta \geq 0,\left|\gamma_{i}\right| \leq 1 \\
& \text { - } \alpha_{i} \geq 0, i=1, \ldots, p, \beta_{j} \geq 0, j=1, \ldots, q \\
& \text { - } 0 \leq \sum_{i=1}^{p} \alpha_{i}+\sum_{j=1}^{q} \beta_{i} \leq 1 \text {, caso contrário. }
\end{aligned}
$$

O parâmetro $\omega$ corresponde ao nível médio da volatilidade da variância condicional, $\delta$ possibilita a estimação de outras potências para o desvio padrão condicional, por meio de uma transformação Box-Cox em $\sigma_{t}$. O coeficiente $\alpha_{i}$ indica a reação da volatilidade a um choque na série e $\beta_{j}$ mede o quanto da volatilidade do período anterior persiste no período corrente. 
A soma de $\alpha_{i}$ e $\beta_{i}$ retrata a persistência da volatilidade, sujeita a restrição dada anteriormente. Caso $\sum\left(\alpha_{i}+\beta_{j}\right)>1$ a série apresenta alta persistência na volatilidade, ou seja, choques na volatilidade perdurarão por um período muito longo na série. A presença do efeito de alavancagem é indicada se $\gamma_{i}>0$. O termo $\mathscr{D}_{v}$ detecta, se existir, a distribuição com cauda pesada e indica a distribuição dos erros com média 0 e variância 1 , em que se $v=0$ trata-se de uma distribuição normal.

A média condicional $\mu$ aqui é modelada por um processo autoregressivo integrado de médias móveis (ARIMA), por meio da metodologia Box e Jenkins (1970). Denota-se por $\operatorname{ARMA}(p, q)$ um processo autorregressivo e de médias móveis de ordem $(p, q)$ que pode ser especificado conforme descrito na equação (7)

$$
r_{t}=\sum_{i=1}^{p} \phi_{i} r_{t-i}+\sum_{i=1}^{q} \theta_{i} \varepsilon_{t-i}+\varepsilon_{t},
$$

em que o termo $\varepsilon_{t}$ é o resíduo que constitui variáveis aleatórias independentes e identicamente distribuídas (iid) com média zero e variância $\sigma^{2}, \phi_{i}, \theta_{j} \in \mathbb{R}$, com $i=1, \ldots, p$ e $j=1, \ldots, q$. A partir da definição do modelo ARMA, na hipótese da série temporal estacionária temos anolagamente um processo $\operatorname{ARIMA}(p, 0, d)$, caso contrário, $r_{t}$ deve ser diferenciada e ajusta-se o $\operatorname{ARIMA}(p, d, q)$ sendo $d$ a ordem de diferenciação da série. A análise da estacionalidade em cada série a ser estimada é realizada com o teste Augmented Dickey-Fuller (ADF teste) proposto por Dickey e Fuller (1979, 1981).

Com as séries de detalhes representando $r_{t}$ em diferentes frequências $j$, os modelos ARIMA-APARCH são estimados com $\widetilde{d}_{j, t}$ usando uma abordagem de máxima verossimilhança (ML). Depois de identificado e estimado o modelo $\operatorname{ARIMA}(p, d, q)$, para modelagem da variância condicional é necessário fazer uma suposição adicional da função densidade $\mathscr{D}_{v}$, denotado $g\left(z_{t} ; \tau\right)$, onde $\tau$ é um vetor de parâmetros da distribuição. A função de log-verossimilhança é dada pela equação (8)

$$
l(\theta, \tau)=\sum_{t=1}^{T} \log f_{j}\left(\tilde{d}_{j, t} \mid \theta, \tau, I_{t-1}\right),
$$

com o estimador de máxima verossimilhança (MLE), $\hat{\theta}_{h M L E}=\max _{\theta \in \Theta} l(\theta, \tau)$ em que $\theta$ é o vetor de parâmetros desconhecidos para a média e variância condicional, $I_{t-1}$ são as informações definidas no tempo $t$.

Uma vez que as séries temporais financeiras frequentemente exibem padrões de não normalidade, apresentando excesso de curtose e assimetria, uma distribuição mais apropriada para $z_{t}$ é a t-Skewed $(v, \xi)$, com os parâmetros $v$ e $\xi$ representando os graus de liberdade e assimetria, respectivamente. Para estrutura GARCH, Lambert e Laurent (2001) especificam a função $l(\theta, \tau)$ dada distribuição t-Skewed, descrita pela equação (9)

$$
\begin{aligned}
l(\theta, v, \xi) & =\mathrm{T}\left[\log \Gamma\left(\frac{v+1}{2}\right)-\log \left(\frac{v}{2}\right)-\frac{1}{2} \log (\pi(v-2))\right] \\
& +\mathrm{T}\left[\log \left(\frac{2}{\xi+\frac{1}{\xi}}\right)+\log (\mathrm{s})\right]-\frac{1}{2} \sum_{t=1}^{T}\left[\log \left(\sigma_{\mathrm{t}}^{2}\right)\right] \\
& -\frac{1}{2} \sum_{t=1}^{T}\left[(1+v) \log \left(1+\frac{\left(s \mathrm{sz}_{t}+m\right)^{2}}{v-2} \xi^{-2 I_{t}}\right)\right],
\end{aligned}
$$

em que

$$
\begin{gathered}
I_{t}= \begin{cases}1, & \text { if } \quad z_{t} \geq-\frac{m}{s}, \\
-1 & \text { if } \quad z_{t}<-\frac{m}{s},\end{cases} \\
m=\frac{\Gamma((v+1 / 2)) \sqrt{v-2}}{\sqrt{\pi} \Gamma(v / 2)}\left(\xi-\frac{1}{\xi}\right) \\
s=\sqrt{\left(\xi^{2}+\frac{1}{\xi^{2}}-1\right)-m^{2} .}
\end{gathered}
$$

\section{Resultados e discussões}

\section{Dados e estatísticas descritivas}

Nas Figuras 1(a) e 1(b) tem-se os gráficos dos preços e dos log-retornos intradiários $(\Delta=15 \mathrm{~min})$, no período de 17 de março a 11 de setembro de 2020. Evidencia-se que os movimentos da série de preços refletem o comportamento intradiário e dos log-retornos a característica de agrupamentos de volatilidade. Estes comportamentos refletem os impactos do COVID-19 nos mercados financeiros mundiais, o qual teve choque inicial dado em março/2020. Em conjunto ao fenômeno, há a influência das instabilidades da política brasileira no período. Também é necessário levar em consideração a expansão que vem apresentado o mercado de capitais brasileiro no campo dos investimentos. Com a análise descritiva, peculiaridades são observadas como assimetria negativa com valor de $-0,65$ e excesso de curtose igual a 40,14. Pela estatística $K S=0,49$ do teste Kolmogorov-Smirnov constata-se também a não normalidade.

O processo de decomposição com a MODWT foi aplicado nas séries usando os procedimentos metodológicos da seção anterior, em que obteve-se $J=11$ níveis de decomposição. Para o ajuste do APARCH, utilizou-se as séries wavelet de detalhe $\widetilde{d}_{j, t}, j=1,3,6,8,10$ referentes as frequências de variação de curtíssimo prazo (ruído estocástico) a longo/médio prazo. As séries obtidas são ilustradas nas Figuras 2(a)-(e). 
Figura 1 - Séries Ibovespa intradiário no período de 17 de março a 11 de setembro $(\Delta=15 \mathrm{~min})$ : (a) Série dos preços; (b) Séries de log-retornos.

(a)

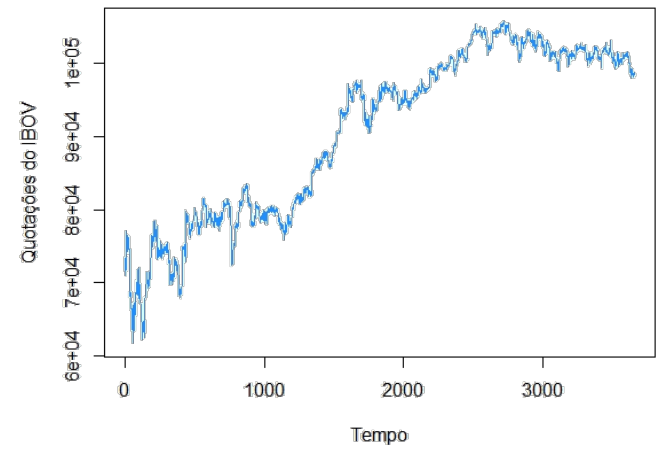

(b)

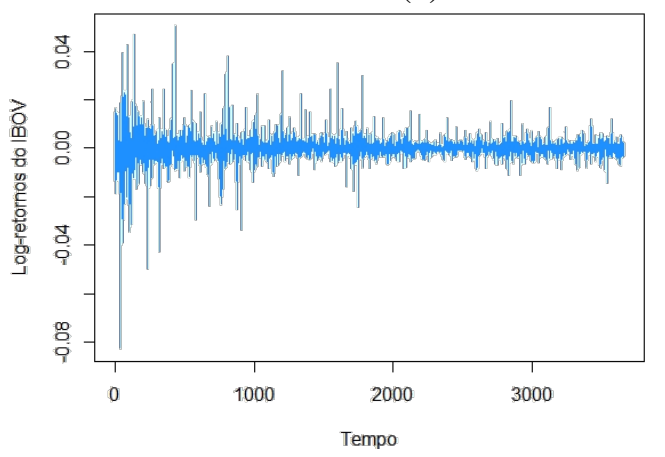

Fonte: Os autores.

Figura 2 - Séries de detalhes $\widetilde{d}_{j, t}$, com $j=1,3,6,8,10$, da decomposição MODWT no log-retornos intradiários do Ibovespa no período de 17 de março a 11 de setembro $(\Delta=15 \mathrm{~min})$.

(a)

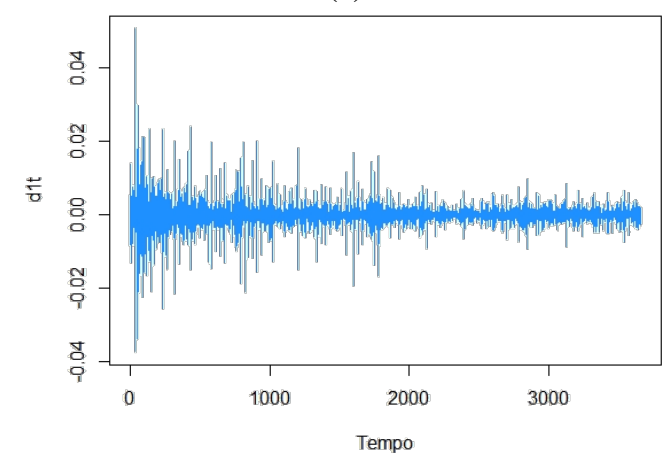

(c)

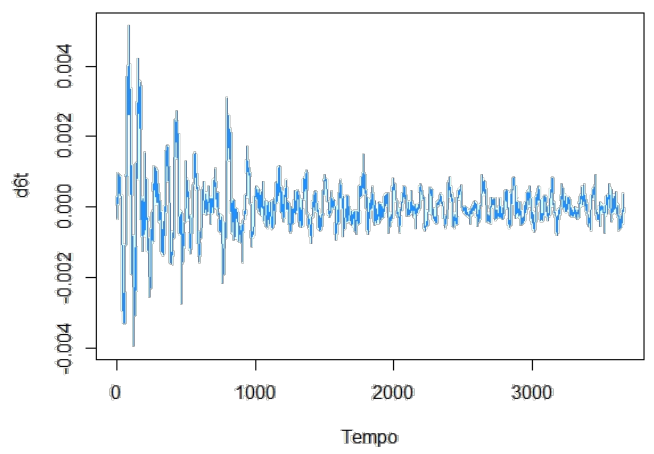

(b)

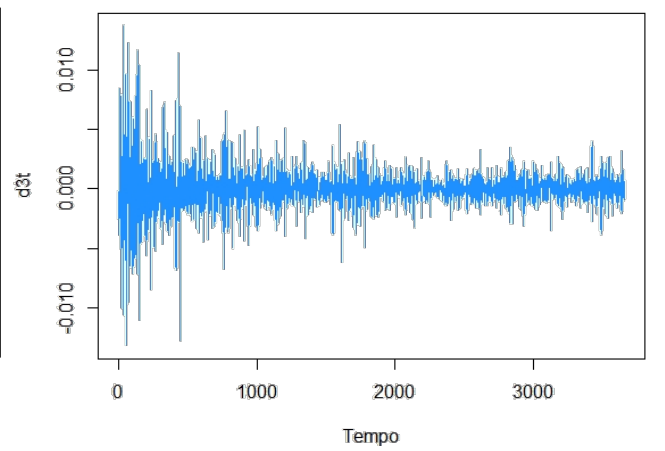

(d)

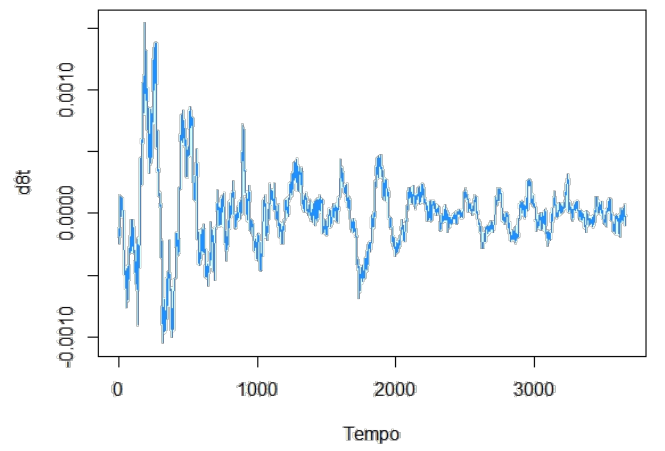

(e)

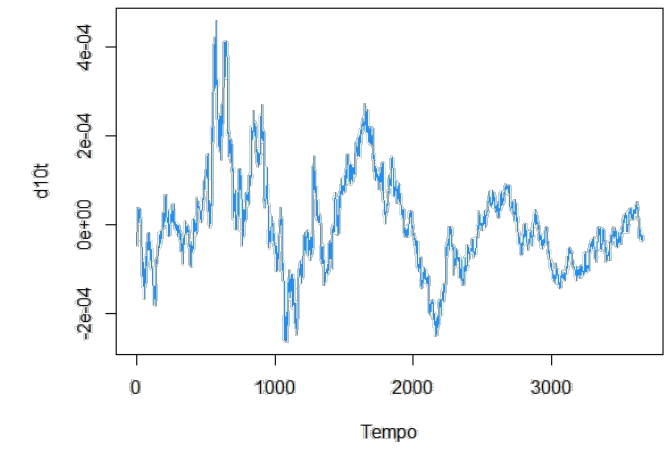

Fonte: Os autores.

Para série $\widetilde{d}_{8, t}$ foi captado o efeito sexta-feira. Assim, dológicos, com base na estimação das equações (4) e (5). foi utilizada a série filtrada na modelagem da volatilidade conforme o método apresentado nos procedimentos metoOs resultados da regressão, com os erros-padrão entre parênteses, são apresentados na Tabela 3 . 
Tabela 3 - Coeficientes de regressão para efeito do dia da semana para $d_{8, t}$.

\begin{tabular}{cc}
\hline Coeficientes & Estimativas \\
\hline$\alpha_{1}$ & 0,000063 \\
& $(0,00001)$ \\
$\alpha_{2}$ & 0,000016 \\
& $(0,00001)$ \\
$\alpha_{3}$ & $-0,00000014$ \\
& $(0,00001)$ \\
$\alpha_{4}$ & 0,000017 \\
& $(0,00001)$ \\
$\alpha_{5}$ & $-0,000039 *$ \\
& $(0,00001)$ \\
\hline *significância de 5\% &
\end{tabular}

Fonte: Os autores.

\section{Análise da Volatilidade}

Definidas as séries obtidas pelos coeficientes wavelets, a condição de estacionariedade foi analisada. Conforme se observa na Tabela 4, os resultados do teste ADF sem constante (sem cte) e constante e tendência (cte+tend) identificaram que apenas a série $\widetilde{d}_{10, t}$ é não estacionária.

Tabela 4 - Teste ADF de raiz unitária para as séries de detalhes $\widetilde{d}_{j, t}, j=1,3,6,8,10$.

\begin{tabular}{ccc}
\hline Séries & Opção & $p$-Valor \\
\hline$\widetilde{d}_{1, t}$ & sem cte & $\leq 0,01$ \\
& cte+tend & $\leq 0,01$ \\
$\widetilde{d}_{3, t}$ & sem cte & $\leq 0,01$ \\
& cte+tend & $\leq 0,01$ \\
$\widetilde{d}_{3, t}$ & sem cte & $\leq 0,01$ \\
& cte+tend & $\leq 0,01$ \\
$\widetilde{d}_{6, t}$ & sem cte & $\leq 0,01$ \\
& cte+tend & $\leq 0,01$ \\
$\widetilde{d}_{8, t}$ & sem cte & $\leq 0,01$ \\
& cte+tend & $\leq 0,01$ \\
$\tilde{d}_{10, t}$ & sem cte & $\leq 0,01$ \\
& cte+tend & 0,04 \\
\hline
\end{tabular}

Fonte: Os autores.

Procedeu-se a estimação dos processos $\operatorname{ARIMA}(p, d, q)$-APARCH$(1,1)$. As especificações para média condicional foram dadas pelos modelos $\operatorname{ARIMA}(1,0,1), \quad \operatorname{ARIMA}(0,0,8), \quad \operatorname{ARIMA}(2,0,0)$, $\operatorname{ARIMA}(1,0,0)$ e $\operatorname{ARIMA}(2,1,1)$ para $\widetilde{d}_{1, t}, \widetilde{d}_{3, t}, \widetilde{d}_{6, t}$, $\widetilde{d}_{8, t}$ filtrada e $\widetilde{d}_{10, t}$, respectivamente. Os resultados das estimativas dos parâmetros, com os erros-padrão entre parênteses, são apresentados na Tabela 5. A estatística Box-Pierce $Q(20)$ dos resíduos padronizados indica a adequabilidade do ajuste dos modelos.

Em relação aos resultados para $\mu$, com os valores estimados das variáveis explicativas definidas por $\phi$ e $\theta$, destaca-se que para a frequência representada pela sé- rie $\widetilde{d}_{3, t}$, Figura 2(b), os resultados refletem a persistência dos movimentos dos preços durante o dia como aponta Schulmeister (2009). Para $\widetilde{d}_{10, t}$, Figura 2(e), foi necessário o ajuste de um $\operatorname{ARIMA}(p, 1, q)$, pois esse nível reflete os movimentos de frequências menores, referente a tendência de médio-longo prazo, que retrata o dinamismo das atividades de negociação de ativos no mercado financeiro. Esse comportamento é evidenciado pelos resultados do teste ADF e com o correlograma apresentado na Figura 3, em que os valores da autocorrelação decaem lentamente para zero indicando a tendência da série.

Em todas as escalas, os resultados para $\sigma$ mostram uma baixa volatilidade incondicional dado pela estimativas de $\omega$. O parâmetro de assimetria $\xi$ foi significativo e positivo, e a estimativa de $v$ captou a presença de caudas pesadas. Os coeficientes $\alpha_{1}$ e $\beta_{1}$ também apresentaram-se significativos para todas as séries. Efeitos assimétricos e de alavancagem na volatilidade são verificados apenas nas decomposições de oscilações de curto prazo e ruídos estocásticos.

Os baixos valores obtidos para $\alpha_{1}$, exceto para frequência semanal, indicam que de forma geral os choques que persistem na volatilidade, dados por $\beta_{1}$, são percebidos rapidamente. No período analisado, os choques se referem, principalmente, as incertezas existentes nas economias mundiais devido aos desdobramentos da pandemia do COVID-19.

Para as frequências intradiárias, diária e mensal mais de $90 \%$ de um choque gerado na série no tempo $t-1$ persistirá no tempo $t$, e para a frequência semanal esse valor ficou em torno de $78 \%$. Adicionalmente, em todas as frequências $\sum \alpha_{1}+\sum \beta_{1}>1$, o que retrata que choques na volatilidade permanecem na série por um período maior de tempo em todas as frequências, ou seja, após um choque, o processo de reversão da variância condicional para seu valor médio tende a ser lento.

$\mathrm{O}$ efeito de alavancagem retratado por $\gamma_{1}>0$ foi evidenciado apenas para os níveis $\tilde{d}_{1, t}$ e $\tilde{d}_{3, t}$, indicando que choques negativos passados, como efeitos do COVID-19, instabilidades econômicas/políticas e modificações exógenas no volume de transações, impactam de forma mais intensa na volatilidade condicional no tempo $t$ do que os choques positivos passados. Em $\tilde{d}_{6, t} \gamma_{1}<0$ o que indica presença de assimetria, sendo que choques positivos impactam mais que os negativos na volatilidade. Para as demais séries $\gamma_{1}$ não foi significativo, apontando que quedas ou altas nos preços podem ter o mesmo efeito sobre a volatilidade. 
Tabela 5 - Estimativas dos modelos ARIMA-APARCH para as séries de detalhes $\widetilde{d}_{j, t}, j=1,3,6,8,10$

\begin{tabular}{|c|c|c|c|c|c|c|c|c|c|c|c|}
\hline Coefficientes & $\widetilde{d}_{1 . t}$ & $\widetilde{d_{3 . t}}$ & $\widetilde{d}_{6 . t}$ & $\widetilde{d}_{8 . t}$ & $\widetilde{d}_{10 . t}$ & Coefficientes & $\widetilde{d}_{1 . t}$ & $\widetilde{d}_{3 . t}$ & $\widetilde{d}_{6 . t}$ & $\widetilde{d_{8 . t}}$ & $\widetilde{d}_{10 . t}$ \\
\hline$\phi_{1}$ & $\begin{array}{c}-0,02^{* *} \\
(0,01)\end{array}$ & & $\begin{array}{l}0,99 * \\
(0,01)\end{array}$ & $\begin{array}{l}0,98^{*} \\
(0,00)\end{array}$ & $\begin{array}{l}0,30 * \\
(0,02)\end{array}$ & $\omega$ & $\begin{array}{l}5 \mathrm{e}^{-5 *} \\
(0,00)\end{array}$ & $\begin{array}{l}1 \mathrm{e}^{-6 *} \\
(0,00)\end{array}$ & $\begin{array}{l}3 \mathrm{e}^{-8 *} \\
(0,00)\end{array}$ & $\begin{array}{l}7 e^{-4 *} \\
(0,00)\end{array}$ & $\begin{array}{l}6 \mathrm{e}^{-8 *} \\
(0,00)\end{array}$ \\
\hline$\phi_{2}$ & & & $\begin{array}{c}-0,03 * \\
(0,01)\end{array}$ & & $\begin{array}{c}-0,02 * * \\
(0,00)\end{array}$ & $\alpha_{1}$ & $\begin{array}{l}0,10^{*} \\
(0,02)\end{array}$ & $\begin{array}{l}0,11^{*} \\
(0,00)\end{array}$ & $\begin{array}{l}0,05^{*} \\
(0,01)\end{array}$ & $\begin{array}{l}0,56^{*} \\
(0,07)\end{array}$ & $\begin{array}{l}0,04 * \\
(0,00)\end{array}$ \\
\hline$\theta_{1}$ & $\begin{array}{c}-0,99^{*} \\
(0,00)\end{array}$ & $\begin{array}{l}0,95^{*} \\
(0,00)\end{array}$ & & & $\begin{array}{c}-0,29^{*} \\
(0,02)\end{array}$ & $\gamma_{1}$ & $\begin{array}{l}0,31 * \\
(0,08)\end{array}$ & $\begin{array}{l}0,28 * \\
(0,07)\end{array}$ & $\begin{array}{c}-1,32 * * \\
(0,07)\end{array}$ & $\begin{array}{l}-0,06 \\
(0,05)\end{array}$ & $\begin{array}{l}-1,21 \\
(0,11)\end{array}$ \\
\hline$\theta_{2}$ & & $\begin{array}{l}0,89^{*} \\
(0,00)\end{array}$ & & & & $\beta_{1}$ & $\begin{array}{c}0,93 * \\
(0,014)\end{array}$ & $\begin{array}{l}0,91^{*} \\
(0,01)\end{array}$ & $\begin{array}{l}0,95^{*} \\
(0,00)\end{array}$ & $\begin{array}{l}0,78^{*} \\
(0,01)\end{array}$ & $\begin{array}{l}0,96 * \\
(0,00)\end{array}$ \\
\hline$\theta_{3}$ & & $\begin{array}{l}0,85^{*} \\
(0,00)\end{array}$ & & & & $\delta$ & $\begin{array}{l}1,18^{*} \\
(0,12)\end{array}$ & $\begin{array}{l}1,25^{*} \\
(0,13)\end{array}$ & $\begin{array}{l}1,38^{*} \\
(0,17)\end{array}$ & $\begin{array}{l}0,35^{*} \\
(0,03)\end{array}$ & $\begin{array}{l}0,98^{*} \\
(0,25)\end{array}$ \\
\hline$\theta_{4}$ & & $\begin{array}{c}-1,00^{*} \\
(0,00)\end{array}$ & & & & $\xi$ & $\begin{array}{l}1,01 * \\
(0,01)\end{array}$ & $\begin{array}{l}1,01^{*} \\
(0,01)\end{array}$ & $\begin{array}{l}1,00^{*} \\
(0,01)\end{array}$ & $\begin{array}{l}0,99 * \\
(0,01)\end{array}$ & $\begin{array}{l}1,00 * \\
(0,01)\end{array}$ \\
\hline$\theta_{5}$ & & $\begin{array}{c}-0,92 * \\
(0,00)\end{array}$ & & & & $v$ & $\begin{array}{l}2,50 * \\
(0,11)\end{array}$ & $\begin{array}{l}2,85^{*} \\
(0,15)\end{array}$ & $\begin{array}{l}3,26^{*} \\
(0,18)\end{array}$ & $\begin{array}{l}2,01^{*} \\
(0,01)\end{array}$ & $\begin{array}{l}2,91 * \\
(0,15)\end{array}$ \\
\hline$\theta_{6}$ & & $\begin{array}{c}-0,87 * \\
(0,00)\end{array}$ & & & & $Q(20)$ & 0,55 & 0,12 & 0,30 & 0,99 & 0,38 \\
\hline$\theta_{7}$ & & $\begin{array}{c}-0,83^{*} \\
(0,00)\end{array}$ & & & & & & & & & \\
\hline$\theta_{8}$ & & $\begin{array}{l}0,02 * \\
(0,00)\end{array}$ & & & & & & & & & \\
\hline
\end{tabular}

*significância de 5\%, **significância de $10 \%$.

Fonte: Os autores.

Figura 3 - Gráfico da função de autocorrelação da série de detalhe $\widetilde{d}_{10, t}$, da decomposição MODWT no log-retornos intradiários do Ibovespa no período de 17 de março a 11 de setembro $(\Delta=15 \mathrm{~min})$.

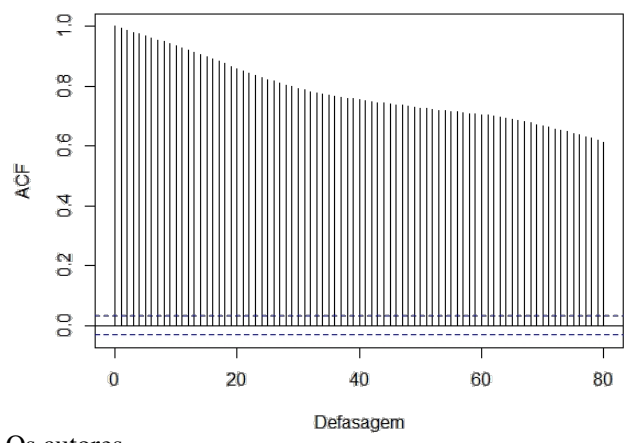

Fonte: Os autores.

Os resultados do coeficiente $\gamma_{1}$ refletem o fato de que os primeiros níveis da decomposição são responsáveis pela maior parte da variabilidade (em torno de $89 \%$ ) de $r_{t}$, como nos trabalhos de Biage (2019) e Kumar e Anandarao (2019). Como decomposições de frequência mais alta foram mais impactantes para detectar o efeito alavancagem, os processos $\operatorname{ARIMA}(p, d, q)$ $\operatorname{APARCH}(1,1)$ foram aplicados na série reconstruída $r_{t}=\widetilde{d}_{1, t}-\widetilde{d}_{3, t}=\sum_{j=1}^{3} \widetilde{d}_{j, t}$ utilizando a equação (3). Na Figura 4 observa-se a reconstrução obtida. Os resultados do teste $\mathrm{ADF}$ não mostraram evidências de raiz unitária na série reconstruída (valor-p $\leq 0,01$ para os casos sem cte e cte+tend). $\mathrm{O}$ modelo $\operatorname{ARIMA}(2,0,1)-\operatorname{APARCH}(1,1)$ foi ajustado, e os resultados são verificados na Tabela 6 , com os erros-padrão entre parênteses, em que assimetria, efeito alavancagem e persistência foram evidenciados no período.
Figura 4 - Série reconstruída com os detalhes $\widetilde{d}_{j, t}$, com $j=1,2,3$, da decomposição MODWT no log-retornos intradiários do Ibovespa $(\Delta=15 \mathrm{~min})$.

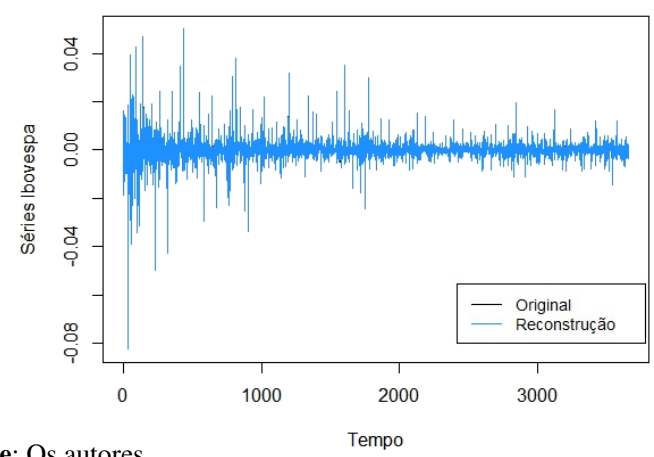

Fonte: Os autores.

Tabela 6 - Estimativas dos modelos ARIMA-APARCH para série reconstruída do Ibovespa intradiário $(\Delta=15 \mathrm{~min})$.

\begin{tabular}{|c|c|c|c|}
\hline Coefficientes & Ibovespa & Coefficientes & Ibovespa \\
\hline$\phi_{1}$ & $\begin{array}{c}-0,630^{*} \\
(0,291)\end{array}$ & $\beta_{1}$ & $\begin{array}{l}0,880 * \\
(0,022)\end{array}$ \\
\hline$\phi_{2}$ & $\begin{array}{c}-0,680 * \\
(0,282)\end{array}$ & $\delta$ & $\begin{array}{l}1,300 * \\
(0,264)\end{array}$ \\
\hline$\theta_{1}$ & $\begin{array}{l}0,656^{*} \\
(0,289)\end{array}$ & $\xi$ & $\begin{array}{l}0,927 * \\
(0,026)\end{array}$ \\
\hline$\omega$ & $\begin{array}{l}0,006^{*} \\
(0,002)\end{array}$ & $v$ & $\begin{array}{l}4,197 * \\
(0,410)\end{array}$ \\
\hline$\alpha_{1}$ & $\begin{array}{l}0,139 * \\
(0,025)\end{array}$ & $Q(20)$ & 0,670 \\
\hline$\gamma_{1}$ & $\begin{array}{l}0,120 * \\
(0,007)\end{array}$ & & \\
\hline
\end{tabular}

*significância de 5\%

Fonte: Os autores. 


\section{Conclusão}

Tendo em vista a dinâmica de ativos financeiros, o objetivo principal deste estudo foi obter o comportamento dos componentes da volatilidade condicional em séries financeiras intradiárias em ciclos de curtissímo, curto, médio e longo prazo. Para isso, utilizamos a técnica MODWT para separar a série dos log-retornos do Ibovespa ( $\Delta=15 \mathrm{~min}$.) em cinco componentes de frequência $\tilde{d}_{j, t}, j=1,3,6,8,10$. Conforme as peculiaridades de séries financeiras intradiárias como assimetria, sazonalidade e agrupamentos de volatilidade, os modelos foram especificados como modelos $\operatorname{ARIMA}(p, d, q)$ $\operatorname{APARCH}(1,1)$ com distribuição t-Skewed $(v, \xi)$.

Aufere-se que no período amostrado, todas as frequências de variação dos retornos do Ibovespa apresentaram baixa volatilidade incondicional e assimetria. Além disso, estão suscetíveis a reações de persistência em sua volatilidade em todas as frequências. Efeitos assimétricos de choques na volatilidade foram significativos para a frequência em torno de 1-2 dias, mas a alavancagem foi captada apenas nas frequências menores (15-120 min.), revelando que oscilações negativas nas cotações têm um efeito maior sobre a volatilidade a curtissímo prazo. A partir das informações auferidas concluímos que impactos das decomposições de alta frequência, componentes que também mostraram efeitos substanciais na série de retornos, refletem a presença do efeito alavancagem. As estimavas obtidas com a modelagem da série reconstruída com os três primeiros níveis da MODWT, corroboram os resultados nas frequências.

Os resultados deste estudo mostram que os modelos estimados com auxílio de recursos multiescala por meio de técnicas wavelet, possibilitam obter informações específicas de determinados períodos sobre a volatilidade intradiária em mercados financeiros. Captar essas informações e seus impactos na variância condicional, principalmente em momentos de incertezas, como os relacionados a influência do avanço da pandemia do COVID-2019 ao redor do mundo, é essencial na elaboração de estratégias de hedge pelos agentes econômicos que podem ser aplicadas tanto no curto como no longo prazo. Logo, as repercussões obtidas podem contribuir na gestão e análise financeira, principalmente as relacionadas a eventos que aumentem a incerteza no mercado acionário brasileiro.

\section{Agradecimentos}

Este estudo foi parcialmente financiado pela Coordenação de Aperfeiçoamento de Pessoal de Nível Superior Brasil (CAPES) - Código Financeiro 001.

\section{Referências}

ALBERG, D.; SHALIT, H.; YOSEF, R. Estimating stock market volatility using asymmetric garch models. Applied Financial Economics, London, v. 18, n. 15, p. 1201-1208, 2008.

AUDRINO, F.; HU, Y. Volatility forecasting: downside risk, jumps and leverage effect. Econometrics, Multidisciplinary Digital Publishing Institute, Basel, v. 4, n. 1, p. 8, 2016.

BAUR, D. G.; DIMPFL, T. Think again: volatility asymmetry and volatility persistence. Studies in Nonlinear Dynamics \& Econometrics, Berlin, v. 23, n. 1, 2018.

BIAGE, M. Analysis of shares frequency components on daily value-at-risk in emerging and developed markets. Physica A: Statistical Mechanics and its Applications, London, v. 532, p. 121798, 2019.

BLACK, F. Studies of stock market volatility changes. In: AMERICAN STATISTICAL ASSOCIATION. Business and Economic Statistics Section, 1976. Proceedings [...]. Washington, DC: American Statistical Association, 1976. p. $171-181$.

BOX, G.; JENKINS, G. Time series analysis: forecasting and control, holden day. San Francisco: Wiley, 1970.

CONSTANTINE, W.; PERCIVAL, D. Wavelet methods for time series analysis. $\mathrm{R}$ package version 2.0-3. Cambridge: Cambridge University Press, 2017.

CROWLEY, P. M. An intuitive guide to wavelets for economists. Journal of Economic Surveys, Clevedon, v. 21, n. 2, p. 207-267, 2007

DALY, K. Financial volatility: Issues and measuring techniques. Physica A: statistical mechanics and its applications, London, v. 387, n. 11, p. 2377-2393, 2008.

DAUBECHIES, I. Ten lectures on wavelets. Philadelphia: Siam, 1992.

DICKEY, D. A.; FULLER, W. A. Distribution of the estimators for autoregressive time series with a unit root. Journal of the American Statistical Association, New York, v. 74, n. 366a, p. 427-431, 1979.

DICKEY, D. A.; FULLER, W. A. Likelihood ratio statistics for autoregressive time series with a unit root. Econometrica, Chicago, p. 1057-1072, 1981. 
DING, Z.; GRANGER, C. W.; ENGLE, R. F. A long memory property of stock market returns and a new model. Journal of Empirical Finance, North-Holland, v. 1, n. 1, p. 83-106, 1993.

ENGLE, R. F. Autoregressive conditional heteroscedasticity with estimates of the variance of United Kingdom inflation. Econometrica: Journal of the econometric society, p. 987-1007, 1982.

GALLEGATI, M.; SEMMLER, W. (ed.). Wavelet applications in economics and finance. Switzerland: Springer, 2014.

HASBROUCK, J. High frequency quoting: short-term volatility in bids and offers. Journal of Financial and Quantitative Analysis, Cambridge, 2016.

IN, F.; KIM, S. An introduction to wavelet theory in finance: a wavelet multiscale approach. Singapore: World scientific, 2013.

JENSEN, M. J.; WHITCHER, B. Measuring the impact intradaily events have on the persistent nature of volatility. In: GALLEGATI, M.; SEMMLER, W. Wavelet applications in economics and finance. New York: Springer, 2014. p. 103-129.

KOVALEVSKY, S. QuantTools: enhanced quantitative trading modelling. R package version 0.5.7. 2018. Disponível em: https://CRAN.R-project.org/package=QuantTools Acesso em: 15 fev. 2019.

KUMAR, A. S.; ANANDARAO, S. Volatility spillover in crypto-currency markets: Some evidences from garch and wavelet analysis. Physica A: Statistical Mechanics and its Applications, London, v. 524, p. 448-458, 2019.

LAMBERT, P.; LAURENT, S. Modelling financial time series using garch-type models and a skewed student density. Liège: Université de Liège, 2001. Mimeo.

LATIF, M.; ARSHAD, S.; FATIMA, M.; FAROOQ, S. Market efficiency, market anomalies, causes, evidences, and some behavioral aspects of market anomalies. Research Journal of Finance and Accounting, [s. l.], v. 2, n. 9, p. 1-13, 2011.

MALLAT, S. Multiresolution approximations and wavelet orthonormal bases of 12(r). Transactions of the American Mathematical Society, New York, v. 315, n. 1, p. 69-87, 1989.

MORETTIN, P. A. Econometria financeira: um curso em séries temporais financeiras. 3. ed. São Paulo: Blucher, 2017.
NAVA, N.; MATTEO, T. D.; ASTE, T. Anomalous volatility scaling in high frequency financial data. Physica $A$ : Statistical Mechanics and its Applications, London, v. 447, p. 434-445, 2016.

OMANE-ADJEPONG, M.; ABABIO, K. A.; ALAGIDEDE, P. Time-frequency analysis of behaviourally classified financial asset markets. Research in International Business and Finance, [s .l.], 2019.

PAN, Z.; LIU, L. Forecasting stock return volatility: A comparison between the roles of short-term and long-term leverage effects. Physica A: Statistical Mechanics and its Applications, London, v. 492, p. 168-180, 2018.

PATTON, A. J.; SHEPPARD, K. Good volatility, bad volatility: Signed jumps and the persistence of volatility. Review of Economics and Statistics, Cambridge, v. 97, n. 3, p. 683-697, 2015.

PERCIVAL, D. B.; WALDEN, A. T. Wavelet methods for time series analysis. Cambridge: Cambridge University Press, 2000. v. 4.

R CORE TEAM. R: A language and environment for statistical computing. 2020. Disponível em: https://www.Rproject.org/. Acesso em: 15 de ago. 2019.

RAMZAN, S.; RAMZAN, S.; ZAHID, F. M. Modeling and forecasting exchange rate dynamics in pakistan using arch family of models. Electronic Journal of Applied Statistical Analysis, Lecce, v. 5, n. 1, p. 15-29, 2012.

ROSSI, M. The efficient market hypothesis and calendar anomalies: a literature review. International Journal of Managerial and Financial Accounting, Genebra, v. 7, n.3-4, p. 285-296, 2015.

SCHULMEISTER, S. Profitability of technical stock trading: Has it moved from daily to intraday data? $R e$ view of Financial Economics, New Orleans, v. 18, n. 4, p. 190-201, 2009.

SHAH, A.; TALI, A.; FAROOQ, Q. Beta through the prism of wavelets. Financial Innovation, London, v. 4, n. 1, p. 18, 2018.

WUERTZ, D.; SETZ, T.; CHALABI, Y.; BOUDT, C.; CHAUSSE, P.; MIKLOVAC, M. fGarch: Rmetrics: autoregressive conditional heteroskedastic modelling. R package version 3042.83.1. 2019. Disponível em: https://CRAN.Rproject.org/package=fGarch. Acesso em: 15 de ago. 2019.

Recebido: 30 set., 2020

Aceito: 23 abril, 2021

Publicado: 30 abril, 2021 